\title{
Diffusioosmosis of electrolyte solutions in a capillary slit with surface charge layers
}

\author{
Hsien Chen Ma, Huan J. Keh* \\ Department of Chemical Engineering, National Taiwan University, Taipei 106-17, Taiwan, ROC
}

Available online 25 July 2005

\begin{abstract}
The steady diffusioosmotic flow of an electrolyte solution in a fine capillary slit with each of its inside walls covered by a layer of adsorbed polyelectrolytes is analytically studied. In this solvent-permeable and ion-penetrable surface charge layer, idealized polyelectrolyte segments are assumed to distribute at a uniform density. The electric double layer and the surface charge layer may have arbitrary thicknesses relative to the gap width between the slit walls. The Debye-Huckel approximation is used to obtain the electrostatic potential distribution on a cross section of the slit. The macroscopic electric field induced by the imposed electrolyte concentration gradient through the slit is determined as a function of the lateral position rather than taken as its constant bulk-phase quantity. Explicit formulas for the fluid velocity profile are derived as the solution of a modified Navier-Stokes/Brinkman equation. The effect of the lateral distribution of the induced axial electric field in the slit on the diffusioosmotic flow is found to be of dominant significance in most practical situations and to drive the fluid towards the end of higher electrolyte concentration. The existence of the surface charge layers can lead to a quite different diffusioosmotic flow from that in a capillary with bare walls.
\end{abstract}

(C) 2005 Elsevier B.V. All rights reserved.

Keywords: Diffusioosmosis; Polyelectrolyte-coated capillary; Arbitrary double-layer thickness

\section{Introduction}

The flow of electrolyte solutions in a small pore with a charged wall is of much fundamental and practical interest in various areas of science and engineering. In general, driving forces for this electrokinetic flow include dynamic pressure differences between the two ends of the pore (a streaming potential is developed as a result of zero net electric current) and tangential electric fields that interact with the electric double layer adjacent to the pore wall (electroosmosis). Problems of fluid flow in pores caused by these well-known driving forces were studied extensively in the past century [1-8].

Another driving force for the electrokinetic flow in a micropore, which has commanded less attention, involves tangential concentration gradients of an ionic solute that interacts with the charged pore wall. This solute-wall interaction is electrostatic in nature and its range is the Debye screening

\footnotetext{
* Corresponding author. Tel.: +886 2 33663048; fax: +886223623040.

E-mail address: huan@ntu.edu.tw (H.J. Keh).
}

length $\kappa^{-1}$ (defined right after Eq. (3)). The fluid motion associated with this mechanism, known as "diffusioosmosis", has been analytically examined for solutions near a plane wall [4,9-12] and inside a fine capillary [13-16]. Electrolyte solutions with a concentration gradient of order $100 \mathrm{kmol} / \mathrm{m}^{4}$ $(=1 \mathrm{M} / \mathrm{cm})$ along solid surfaces with a zeta potential of order $k T / e(\sim 25 \mathrm{mV} ; e$ is the charge of a proton, $k$ is the Boltzmann constant, and $T$ is the absolute temperature) can flow by diffusioosmosis at velocities of several micrometers per second.

Although the basic relationships involved in electrokinetic phenomena were derived mainly by using the traditional model of plain distribution of surface charges, quite a number of investigations have applied these phenomena to the study of the effects of polyelectrolyte adsorbates. The electroosmotic flows in capillaries with thin polymer layers on the inside walls were theoretically examined for the cases of a slit $[17,18]$ and a tube [19] with thin double layers. On the other hand, analytical formulas for the electroosmotic velocity profile of electrolyte solutions on the cross section of a capillary with its inside wall covered by a finite layer 
of adsorbed polyelectrolytes were obtained by solving the linearized Poisson-Boltzmann equation for the case of an arbitrary value of $\kappa R$ or $\kappa h$, where $R$ is the radius of a capillary tube and $h$ is the half thickness of a capillary slit [20,21]. Recently, the diffusioosmotic flow of a symmetric electrolyte solution in a fine capillary slit bearing adsorbed polyelectrolytes on its inside walls was theoretically investigated for the case of small potentials or low fixed-charge densities, and an analytical formula for the fluid velocity profile on the cross section of the slit was obtained [22]. In this study, however, the effect of lateral distributions of the counter-ions and co-ions on the local macroscopic electric field induced by the imposed electrolyte concentration gradient in the axial direction, which can be dominantly important, was neglected.

The objective of this work is to analyze the steady diffusioosmosis of an electrolyte solution with a constant imposed concentration gradient through a capillary slit bearing permanently adsorbed or covalently bound polyelectrolytes on its inside walls. The charge and segment densities of the adsorbed polymers are assumed to be uniform throughout the surface charge layer, and the Debye-Huckel approximation for the electrostatic potential is employed. However, no assumptions will be made about the thickness of the electric double layer or the thickness of the surface charge layer relative to the gap width between the slit walls, and the lateral distribution of the induced axial electric field is allowed. We shall derive explicit formulas for the local and average fluid velocities due to the application of an electrolyte concentration gradient along the slit walls. These results show that the effect of the deviation of the induced axial electric field in the slit from its bulk-phase quantity on the diffusioosmotic velocity of the fluid is dominantly significant in most practical situations.

\section{Electrostatic potential distribution}

In this section, we consider the electrostatic potential distribution in the fluid solution of a symmetrically charged electrolyte on a cross section of the narrow channel between two large identical parallel plates of length $L$ at separation distance $2 h$ with $h \ll L$, as illustrated in Fig. 1. Each of the inside walls of the capillary slit is coated with a layer of adsorbed,

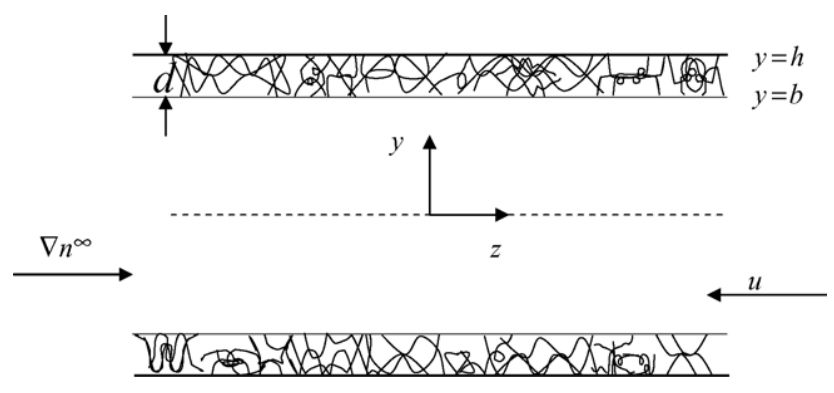

Fig. 1. Geometrical sketch for the diffusioosmosis in a capillary slit with each of its inside walls covered by a layer of adsorbed polyelectrolytes. charged polymers in equilibrium with the surrounding solution. The polymer layer is treated as a solvent-permeable and ion-penetrable surface charge layer of constant thickness $d=h-b$ in which fixed-charged groups of valence $q$ are distributed at a uniform density $N$. Experimental values for human erythrocytes [23], rat lymphocytes [24], and grafted polymer macrocapsules [25] indicate that $d$ ranges from $7.8 \mathrm{~nm}$ to $3.38 \mu \mathrm{m}$ and $N$ can be as high as $0.03 \mathrm{kmol} / \mathrm{m}^{3}$, depending on the $\mathrm{pH}$ and ionic strength of the electrolyte solution.

The prescribed electrolyte concentration gradient $\nabla n^{\infty}$ is a constant along the axial direction in the capillary, where $n^{\infty}(z)$ can be interpreted as the equivalent electrolyte concentration in the bulk solution phase (outside the capillary) at equilibrium with the fluid in the slit cross section at axial position $z$. Since the electrolyte ions can diffuse freely along the capillary (inside and outside the surface charge layers), there exists no regular osmotic flow of the solvent. It is assumed that $L\left|\nabla n^{\infty}\right| / n^{\infty}(0) \ll 1$, where $z=0$ is set at the midpoint through the capillary slit. Thus, the variation of the electrostatic potential (excluding the macroscopic electric field induced by the prescribed electrolyte gradient, which will be discussed in the next section) and ionic concentrations in the slit with the axial position is negligible.

Because of the planar symmetry of the system, we need consider only the half region $0 \leq y \leq h$, where $y$ is the distance from the median plane between the slit walls in a normal direction. If $\psi(y)$ represents the electrostatic potential at the position $y$ relative to that in the bulk solution and $n_{+}(y, z)$ and $n_{-}(y, z)$ denote the local concentrations of the cation and anion, respectively, of the symmetric electrolyte with valence $Z$ (which is positive), then Poisson's equation gives

$\frac{\mathrm{d}^{2} \psi}{\mathrm{d} y^{2}}=-\frac{4 \pi e}{\varepsilon}\left\{Z\left[n_{+}(y, 0)-n_{-}(y, 0)\right]+H(y) q N\right\}$

Here, $H(y)$ is a unit step function which equals unity if $b<y<h$, and zero if $0 \leq y<b ; \varepsilon=4 \pi \varepsilon_{0} \varepsilon_{\mathrm{r}}$, where $\varepsilon_{\mathrm{r}}$ is the relative permittivity of the electrolyte solution which is assumed to be constant and $\varepsilon_{0}$ is the permittivity of a vacuum.

The local concentrations $n_{+}$and $n_{-}$are also related to $\psi$ by the Boltzmann equation:

$n_{ \pm}=n^{\infty} \exp \left(\mp \frac{Z e \psi}{k T}\right)$

The substitution of Eq. (2) into Eq. (1) leads to the wellknown Poisson-Boltzmann equation. For small values of $\psi$ (or Ze $\psi / k T \ll 1$, known as the Debye-Huckel approximation), the Poisson-Boltzmann equation can be linearized and Eq. (1) becomes

$\frac{\mathrm{d}^{2} \psi}{\mathrm{d} y^{2}}=\kappa^{2} \psi-H(y) \frac{4 \pi q e N}{\varepsilon}$,

where $\kappa=\left[8 \pi(Z e)^{2} n^{\infty}(0) / \varepsilon k T\right]^{1 / 2}$ is the Debye screening parameter. 
The boundary conditions for $\psi$ are

$\frac{\mathrm{d} \psi}{\mathrm{d} y}(y=0)=0$,

$\psi\left(y=b^{-}\right)=\psi\left(y=b^{+}\right)$,

$\frac{\mathrm{d} \psi}{\mathrm{d} y}\left(y=b^{-}\right)=\frac{\mathrm{d} \psi}{\mathrm{d} y}\left(y=b^{+}\right)$,

$\frac{\mathrm{d} \psi}{\mathrm{d} y}(y=h)=\frac{4 \pi \sigma}{\varepsilon}$.

Eqs. (4b) and (4c) are the continuity requirements for $\psi$ and $\mathrm{d} \psi / \mathrm{d} y$ at the outer edge of the surface charge layer $[18,26]$. Eq. (4d) is the Gauss condition at the capillary wall, with $\sigma$ equal to the surface charge density of the bare wall.

The solution to Eqs. (3) and (4) is

$\psi=\frac{k T}{Z e} A \cosh (\kappa y), \quad$ if $0 \leq y \leq b$,

$\psi=\frac{k T}{Z e}[B \cosh (\kappa y)+C \sinh (\kappa y)+\bar{N}], \quad$ if $b \leq y \leq h$,

with

$A=\frac{\bar{\sigma}+\bar{N} \sinh (\kappa d)}{\sinh (\kappa h)}$,

$B=\frac{\bar{\sigma}-\bar{N} \cosh (\kappa h) \sinh (\kappa b)}{\sinh (\kappa h)}$,

$C=\bar{N} \sinh (\kappa b)$,

where $\bar{\sigma}=4 \pi Z e \sigma / \varepsilon \kappa k T$ and $\bar{N}=4 \pi Z e^{2} q N / \varepsilon \kappa^{2} k T$. Evidently, the electric potential given by Eq. (5) is correct to the first orders of the dimensionless fixed-charge densities $\bar{\sigma}$ and $\bar{N}$. Note that the parameter $\bar{N}$ can also be viewed as the nondimensionalized Donnan potential $[18,27]$ of the surface charge layer in the Debye-Huckel approximation.

If the boundary condition (4d) for the case of constant surface charge density is replaced by the boundary condition for the case of constant surface potential,

$\psi(y=h)=\zeta$,

then the solution in the form of Eq. (5) is also valid to the first orders of $\bar{\zeta}$ and $\bar{N}$, with

$A=\frac{\bar{\zeta}+\bar{N}[\cosh (\kappa d)-1]}{\cosh (\kappa h)}$,

$B=\frac{\bar{\zeta}-\bar{N}[\sinh (\kappa h) \sinh (\kappa b)+1]}{\cosh (\kappa h)}$,

where $\bar{\zeta}=Z e \zeta / k T$ is the dimensionless surface potential, and $c$ is still given by Eq. (6c). By using Eqs. (4d), (5b), (6c), and (8b), it can be found that the relation between $\bar{\zeta}$ and $\bar{\sigma}$ for arbitrary values of $\bar{N}, \kappa h$ and $\kappa b$ under the Debye-Huckel approximation is

$\bar{\sigma} \cosh (\kappa h)=(\bar{\zeta}-\bar{N}) \sinh (\kappa h)+\bar{N} \sinh (\kappa b)$
When there is no polyelectrolyte adsorbed on the walls of the capillary slit, or when the adsorbed polymer layer is uncharged, one has $N=0$. Then, Eqs. (5), (6), and (8) reduce to

$\psi=\frac{k T}{Z e} A_{0} \cosh (\kappa y)$,

where

$A_{0}=\frac{\bar{\sigma}}{\sinh (\kappa h)}$

for the situation of constant surface charge density, and

$A_{0}=\frac{\bar{\zeta}}{\cosh (\kappa h)}$

for the situation of constant surface potential $\left(B=A=A_{0}\right.$ and $C=0)$. When the capillary slit is filled with the polyelectrolytes, one has $d=h$ and $b=0$. Then, Eqs. (5), (6), and (8) reduce to

$\psi=\frac{k T}{Z e}\left[B_{1} \cosh (\kappa y)+\bar{N}\right]$,

where

$B_{1}=\frac{\bar{\sigma}}{\sinh (\kappa h)}$

for the situation of constant surface charge density, and

$B_{1}=\frac{\bar{\zeta}-\bar{N}}{\cosh (\kappa h)}$

for the situation of constant surface potential $(A-\bar{N}=B=$ $B_{1}$ and $C=0$ ).

\section{Induced electric field distribution}

The ionic concentrations $n_{+}$and $n_{-}$in the capillary slit are not uniform in both axial $(z)$ and lateral $(y)$ directions; hence their prescribed gradients in the axial direction can give rise to a "diffusion current" distribution on a cross section of the slit. To prevent a continuous separation of the counter-ions and co-ions, an electric field distribution $E$ along the axial direction arises spontaneously in the electrolyte solution to produce another electric current distribution, which exactly balances the diffusion current $[4,10]$.

The flux of either ionic species can be expressed by the Nernst-Planck equation,

$\boldsymbol{J}_{ \pm}=-D_{ \pm}\left[\nabla n_{ \pm} \pm \frac{Z e}{k T} n_{ \pm}(\nabla \psi-\boldsymbol{E})\right]$,

where the principle of superposition for the electric potential is used, and $D_{+}$and $D_{-}$are the diffusion coefficients of the cations and anions, respectively, which are assumed to be constant both inside and outside the porous surface layer. In order to have no current arising from the cocurrent diffusion 
of the cations and anions, one must require that $\boldsymbol{J}_{+}=\boldsymbol{J}_{-}=\boldsymbol{J}$. Applying this constraint to Eq. (14), one obtains

$\boldsymbol{E}=\frac{k T}{Z e}\left(\frac{G_{+}-G_{-}}{G_{+}+G_{-}}\right) \frac{\nabla n^{\infty}}{n^{\infty}(0)}$,

where

$G_{ \pm}=D_{ \pm} \exp \left(\mp \frac{Z e \psi}{k T}\right)$

Here, the coefficients $G_{+}$and $G_{-}$reflect the fact of an increase in the axial diffusive flux of the counter-ions and a decrease in the flux of the co-ions inside the electric double layer.

Substitution of Eqs. (15) and (16) into Eq. (14) leads to,

$\boldsymbol{J}=-D \nabla n^{\infty}$,

where the position-dependent net diffusivity is

$D=\frac{2 G_{+} G_{-}}{G_{+}+G_{-}}$

Eqs. (15) and (17) show clearly that both $\boldsymbol{E}$ and $\boldsymbol{J}$ are collinear with the axially imposed electrolyte gradient $\nabla n^{\infty}$.

When the electric potential $\psi$ is low, a Taylor expansion applies to Eq. (16), and Eqs. (15) and (18) can be expressed as

$\boldsymbol{E}=\frac{k T}{Z e}\left[\beta-\left(1-\beta^{2}\right) \frac{Z e \psi}{k T}+\mathrm{O}\left(\psi^{2}\right)\right] \frac{\nabla n^{\infty}}{n^{\infty}(0)}$

and

$D=\frac{2 D_{+} D_{-}}{D_{+}+D_{-}}\left[1+\beta \frac{Z e \psi}{k T}+\mathrm{O}\left(\psi^{2}\right)\right]$,

where

$\beta=\frac{D_{+}-D_{-}}{D_{+}+D_{-}}$

Evidently, $-1 \leq \beta \leq 1$. Note that, even if the cation and anion diffusion coefficients are identical (i.e., $\beta=0$, the $\mathrm{O}(\psi)$ term in Eq. (19) for the induced electric field $\boldsymbol{E}$ still exists (due to the adsorption of the counter-ions and depletion of the co-ions near the slit walls) and equals $-\psi \nabla n^{\infty} / n^{\infty}(0)$. In a previous study of the diffusioosmosis of electrolyte solutions in a capillary slit with each of its inside walls covered by a layer of adsorbed polyelectrolytes [22], only the first term in the brackets of Eq. (19), which is a constant, was considered for $\boldsymbol{E}$ (the bulk-phase electrostatic potential $\psi=0$ is taken everywhere), and thus, the effect of the lateral distribution of the induced electric field on the fluid velocity was excluded.

\section{Fluid velocity distribution}

We now consider the steady flow of an electrolyte solution in a capillary slit with each of its inside walls coated with a layer of charged polymers under the influence of a constant concentration gradient of the electrolyte prescribed axially.
The momentum balances on the Newtonian fluid in the $y$ and $z$ directions give

$\frac{\partial p}{\partial y}+Z e\left(n_{+}-n_{-}\right) \frac{\mathrm{d} \psi}{\mathrm{d} y}=0$

$\eta \frac{\mathrm{d}^{2} u}{\mathrm{~d} y^{2}}-H(y) f u=\frac{\partial p}{\partial z}-Z e\left(n_{+}-n_{-}\right)|\boldsymbol{E}|$

Here, $u(y)$ is the fluid velocity profile (satisfying the equation of continuity for an incompressible fluid) in the direction of decreasing electrolyte concentration (i.e., direction of $\nabla n^{\infty}$ ), $p(y, z)$ is the pressure, $\boldsymbol{E}(y)$ is the macroscopic electric field induced by the applied concentration gradient of the electrolyte given by Eq. (15) or (19), $\eta$ is the viscosity of the fluid, and $f$ is the hydrodynamic friction coefficient in the polymer layer per unit volume of the fluid; both $\eta$ and $f$ are assumed to be constant. Eq. (23) is the Navier-Stokes/Brinkman equation modified by adding a term of electrostatic force.

The boundary conditions for $u$ are

$\frac{\mathrm{d} u}{\mathrm{~d} y}(y=0)=0$,

$u\left(y=b^{-}\right)=u\left(y=b^{+}\right)$,

$\frac{\mathrm{d} u}{\mathrm{~d} y}\left(y=b^{-}\right)=\frac{\mathrm{d} u}{\mathrm{~d} y}\left(y=b^{+}\right)$,

$u(y=h)=0$

Eqs. (24b) and (24c) express the continuity conditions of $u$ and of $\mathrm{d} u / \mathrm{d} y$ at the outer boundary of the surface charge layer [17-19]. In Eq. (24d), we have assumed that the shear plane coincides with the surface of the bare wall.

After the substitution of Eq. (2) for $n_{ \pm}$into Eq. (22) (based on the assumption that the equilibrium lateral ionic distributions are not affected by the axially induced electric field $\boldsymbol{E})$ and the application of the Debye-Huckel approximation, the pressure distribution can be determined, with the result correct to the second orders of $\bar{\sigma}$ (or $\bar{\zeta}$ ) and $\bar{N}$ as

$p=p_{0}+\frac{n^{\infty}(z)}{k T}(Z e)^{2}\left\{[\psi(y)]^{2}-[\psi(0)]^{2}\right\}$

Here, $p_{0}$ is the pressure at the median plane between the slit walls, which is a constant in the absence of applied pressure gradient, and the electric potential distribution $\psi(y)$ is given by Eq. (5).

Substituting the linearized form of the ionic concentration distributions of Eq. (2), the electrostatic potential distribution of Eq. (5), the pressure profile of Eq. (25), and the induced electric field profile of Eq. (19) into Eq. (23), and solving for the fluid velocity subject to the boundary conditions in Eq. (24), we obtain

$\frac{u}{U^{*}}=\beta \Phi_{1}+\frac{1}{8} \Phi_{2}-\frac{1}{4}\left(1-\beta^{2}\right) \Phi_{3}$, 
where

$$
\begin{aligned}
\Phi_{i}= & g_{i 1}(\kappa b)-g_{i 1}(\kappa y)-g_{i 2}(\kappa b)+\operatorname{sech}(\lambda d) g_{i 2}(\kappa h) \\
& -\tanh (\lambda d) g_{i 0}(\kappa b), \quad \text { if } 0 \leq y \leq b,
\end{aligned}
$$

$$
\begin{aligned}
\Phi_{i}= & \operatorname{sech}(\lambda d)\left[g_{i 2}(\kappa h) \cosh (\lambda y-\lambda b)\right. \\
& \left.-g_{i 0}(\kappa b) \sinh (\lambda h-\lambda y)\right]-g_{i 2}(\kappa y), \quad \text { if } b \leq y \leq h,
\end{aligned}
$$

for $i=1,2$, and 3 . In the above equations, $U^{*}=$ $2 k T\left|\nabla n^{\infty}\right| / \eta \kappa^{2}$, which is a characteristic value of the diffusioosmotic velocity, the functions $g_{i 0}(x), g_{i 1}(x)$, and $g_{i 2}(x)$ are defined by Eqs. (A.1)-(A.3) in Appendix A, and $\lambda=(f / n)^{1 / 2}$. The parameter $1 / \lambda$ has the dimension of length and the square of it is the so-called Darcy permeability of the porous medium, which is related to the pore (or segment) size and porosity and characterizes the dynamic behavior of the viscous fluid in it. For the surface charge layers of human erythrocytes [18], rat lymphocytes [24], and grafted polymer microcapsules [25], experimental data of $i / \lambda$ range from 1.35 to $3.7 \mathrm{~nm}$. It can be found from Eq. (27) that the functions $\Phi_{2}$ and $\Phi_{3}$ are almost equivalent when the value of $\kappa h$ is large and the value of $d / h$ is small.

The definition of the average fluid velocity over a cross section of the capillary slit is

$$
\langle u\rangle=\frac{1}{h} \int_{0}^{h} u(y) \mathrm{d} y
$$

Substituting Eqs. (26) and (27) into the above equation and performing the integration, we obtain

$$
\frac{\langle u\rangle}{U^{*}}=\beta\left\langle\Phi_{1}\right\rangle+\frac{1}{8}\left\langle\Phi_{2}\right\rangle-\frac{1}{4}\left(1-\beta^{2}\right)\left\langle\Phi_{3}\right\rangle,
$$

with

$$
\begin{aligned}
\left\langle\Phi_{i}\right\rangle= & \frac{b}{h}\left[g_{i 1}(\kappa b)-g_{i 2}(\kappa b)\right]+\frac{1}{\lambda h}[\lambda b \operatorname{sech}(\lambda d) \\
& +\tanh (\lambda d)] g_{i 2}(\kappa h) \\
& -\frac{1}{\lambda h}[\lambda b \tanh (\lambda d)-\operatorname{sech}(\lambda d)+1] g_{i 0}(\kappa b) \\
& -s_{i 1}(\kappa b)-s_{i 2}(\kappa b),
\end{aligned}
$$

where the functions $S_{i 1}(x)\left(=\int_{0}^{x} g_{i 1}(x) \mathrm{d} x / \kappa h\right)$ and $S_{i 2}(x)$ $\left(=\int_{0}^{x} g_{i 2}(x) \mathrm{d} x / \kappa h\right)$ are defined by Eqs. (A.5) and (A.6).

The function $\Phi_{1}$ in Eqs. (26) and (29) represents the first orders of $\bar{\sigma}($ or $\bar{\zeta})$ and $\bar{N}$, while the functions $\Phi_{2}$ and $\Phi_{3}$ denote the second orders. As it is well-known, the diffusioosmosis of an electrolyte solution in a capillary pore results from a linear combination of two effects: "chemiosmosis" due to the nonuniform adsorption of counter-ions and depletion of coions in the electric double layer over the charged surface and "electroosmosis" due to the macroscopic electric field generated by the imposed concentration gradient of the electrolyte given by Eq. (15) or (19). The terms in Eqs. (26) and (29) involving the functions $\Phi_{1}$ and $\Phi_{3}$ represent the contribution from electroosmosis, while the remainder terms (containing the function $\Phi_{2}$ ) are the chemiosmotic component. Note that additional second-order terms caused by electroosmosis may exist if the electrolyte is not symmetric.

When there is no polymer adsorbed on the slit walls, one has $d=0, b=h, N=0$, and $\lambda=0$ and the potential profile in the slit is given by Eq. (10). Then, Eqs. (27) and (30) reduce to

$$
\begin{aligned}
& \Phi_{i}=g_{i 1}(\kappa h)-g_{i 1}(\kappa y) \\
& \langle\Phi\rangle=g_{i 1}(\kappa h)-s_{i 1}(\kappa h)
\end{aligned}
$$

In the functions $g_{i 1}$ and $S_{i 1}$ for this simple case, we take $A=A_{0}$, which was defined by Eq. (11). Eqs. (31) and (32) agree with the result obtained in a previous article [16], in which only the case of a capillary with bare walls is examined. In this case, the functions $\Phi_{i}$ and $\left\langle\Phi_{i}\right\rangle$ with $i$ equal to 1,2 , and 3 are always positive and $\Phi_{3} \geq \Phi_{2}$. It can be found by a comparison between Eqs. (30) and (32) that the structure of the surface charge layer can result in an augmented or a diminished fluid velocity relative to that in a capillary with bare walls, depending on the characteristics of the electrolyte solution, of the surface charge layer, and of the capillary.

For the case of a capillary slit coated with an uncharged polymer layer $(N=0)$ at each of its inside walls, Eq. (10) for the potential distribution is also applicable and the fluid velocity can be evaluated from Eqs. (26)-(30) by setting $\bar{N}=$ $0, B=A=A_{0}$ and $C=0$.

When $\lambda \rightarrow \infty$ (very high segment density), the resistance to the fluid motion inside the surface charge layer is infinitely large. For this limiting case, $g_{i 0}(x)=g_{i 2}(x)=0$, and Eqs. (27) and (30) reduce to

$$
\begin{aligned}
& \Phi_{i}=g_{i 1}(\kappa b)-g_{i 1}(\kappa y), \quad \text { if } 0 \leq y \leq b, \\
& \Phi_{i}=0, \quad \text { if } b \leq y \leq h, \\
& \left\langle\Phi_{i}\right\rangle=\frac{b}{h} g_{i 1}(\kappa b)-s_{i 1}(\kappa b)
\end{aligned}
$$

Eq. (33) shows that the fluid flow in the surface charge layer disappears and the velocity profile of the remaining fluid is similar to that in a polymer-free capillary slit of half thickness $b$ with a modified surface charge density or surface potential at the wall.

When $\lambda \rightarrow 0$ (very low segment density), the adsorbed polymers do not exert resistance to the fluid motion in the capillary channel. In this limit, Eqs. (27) and (30) become

$$
\begin{aligned}
& \Phi_{i}=g_{i 1}(\kappa b)-g_{i 1}(\kappa y)+g_{i 3}(\kappa b), \quad \text { if } 0 \leq y \leq b, \\
& \Phi_{i}=g_{i 3}(\kappa y), \quad \text { if } b \leq y \leq h ; \\
& \left\langle\Phi_{i}\right\rangle=\frac{b}{h}\left[g_{i 1}(\kappa b)+g_{i 3}(\kappa b)\right]-s_{i 1}(\kappa b)+s_{i 3}(\kappa b),
\end{aligned}
$$

where the functions $g_{i 3}$ and $S_{i 3}(x)\left(=\int_{x}^{\kappa h} g_{i 3}(x) \mathrm{d} x / \kappa h\right)$ are defined by Eqs. (A.7) and (A.8). If the adsorbed polymers 
are uncharged $(N=0)$, the above expressions for the fluid velocity again reduce to Eqs. (31) and (32).

When the capillary slit is filled with the adsorbed polymers, one has $d=h$ and $b=0$, and the potential distribution in the slit is given by Eq. (12). Then, Eqs. (27) and (30) reduce to

$$
\begin{aligned}
& \Phi_{i}=g_{i 4}(\kappa h) \frac{\cosh (\lambda y)}{\cosh (\lambda h)}-g_{i 4}(\kappa y) \\
& \left\langle\Phi_{i}\right\rangle=g_{i 4}(\kappa h) \frac{\tanh (\lambda h)}{\lambda h}-s_{i 4}(\kappa h),
\end{aligned}
$$

where the definitions of the functions $g_{i 4}(x)$ and $S_{i 4}(x)$ $\left(=\int_{0}^{x} g_{i 4}(x) \mathrm{d} x / \kappa h\right)$ are given by Eqs. (A.9) and (A.10).

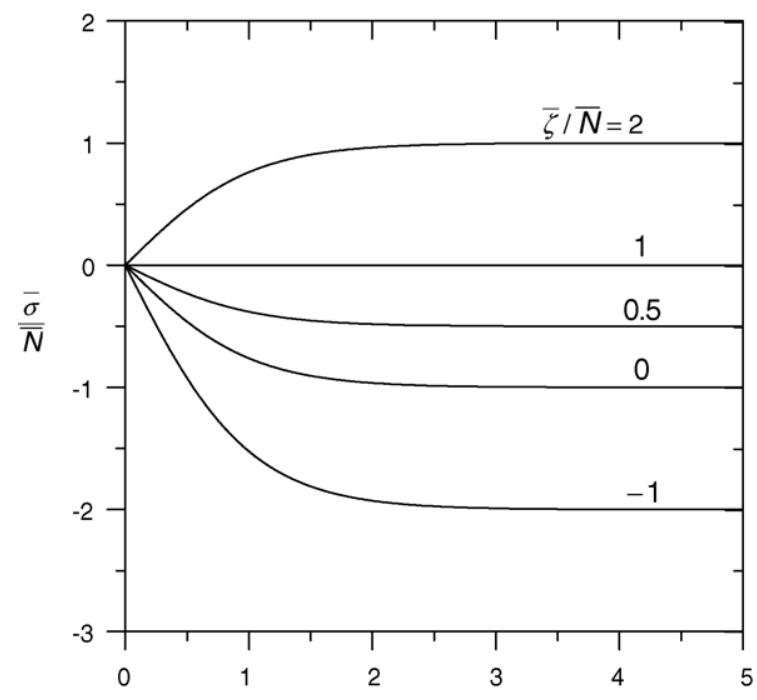

(a)

$\kappa h$

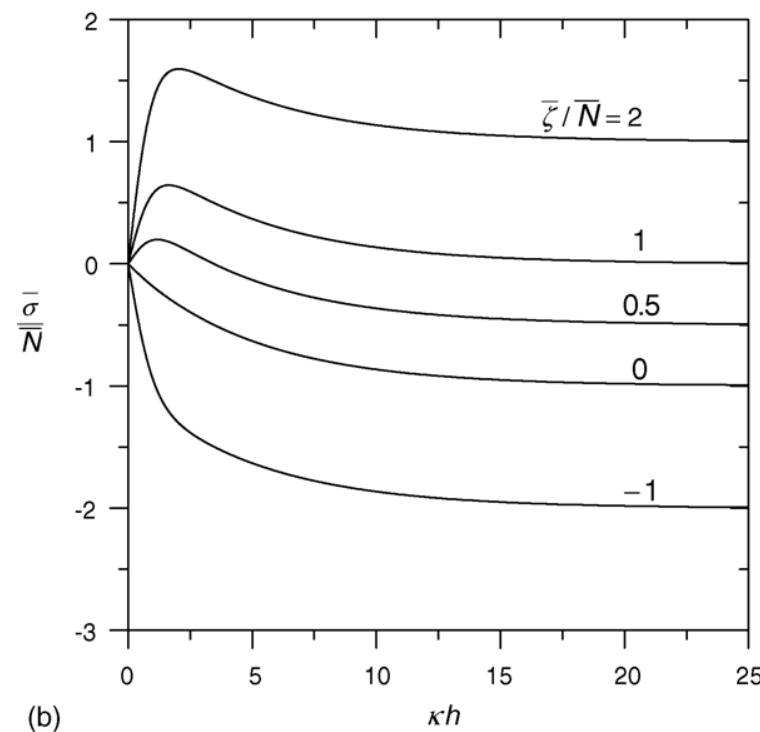

Fig. 2. Plots of the ratio $\bar{\sigma} / \bar{N}$ for a capillary slit with its inside walls covered by layers of adsorbed polyelectrolytes vs. the parameter $\kappa h$ : (a) $b / h=0$; (b) $b / h=0.8$.

\section{Results and discussion}

For the system of an electrolyte solution in a capillary slit bearing adsorbed polyelectrolytes on its inside walls, the surface charge density of the wall, the surface potential of the wall, the fixed-charge density in the polyelectrolyte layer, and the electrokinetic dimensions of the system are related by Eq. (9). Fig. 2(a) and (b) show the results of the ratio $\bar{\sigma} / \bar{N}$ for the case of $b / h=0$ and for the case of a finite value of $b / h(=0.8)$, respectively, as functions of $\kappa h$ for several values of the ratio $\bar{\zeta} / \bar{N}$. It can be seen that $\bar{\sigma} / \bar{N}=0$ as $\kappa h=0$ and $\bar{\sigma} / \bar{N}=\bar{\zeta} / \bar{N}-1$ as $\kappa h \rightarrow \infty$, regardless of the values of $\bar{\zeta} / \bar{N}$ and $b / h$. For the special case with $b / h=0$ and $\bar{\zeta} / \bar{N}=1$, the potential in the polyelectrolyte-filled capillary equals the
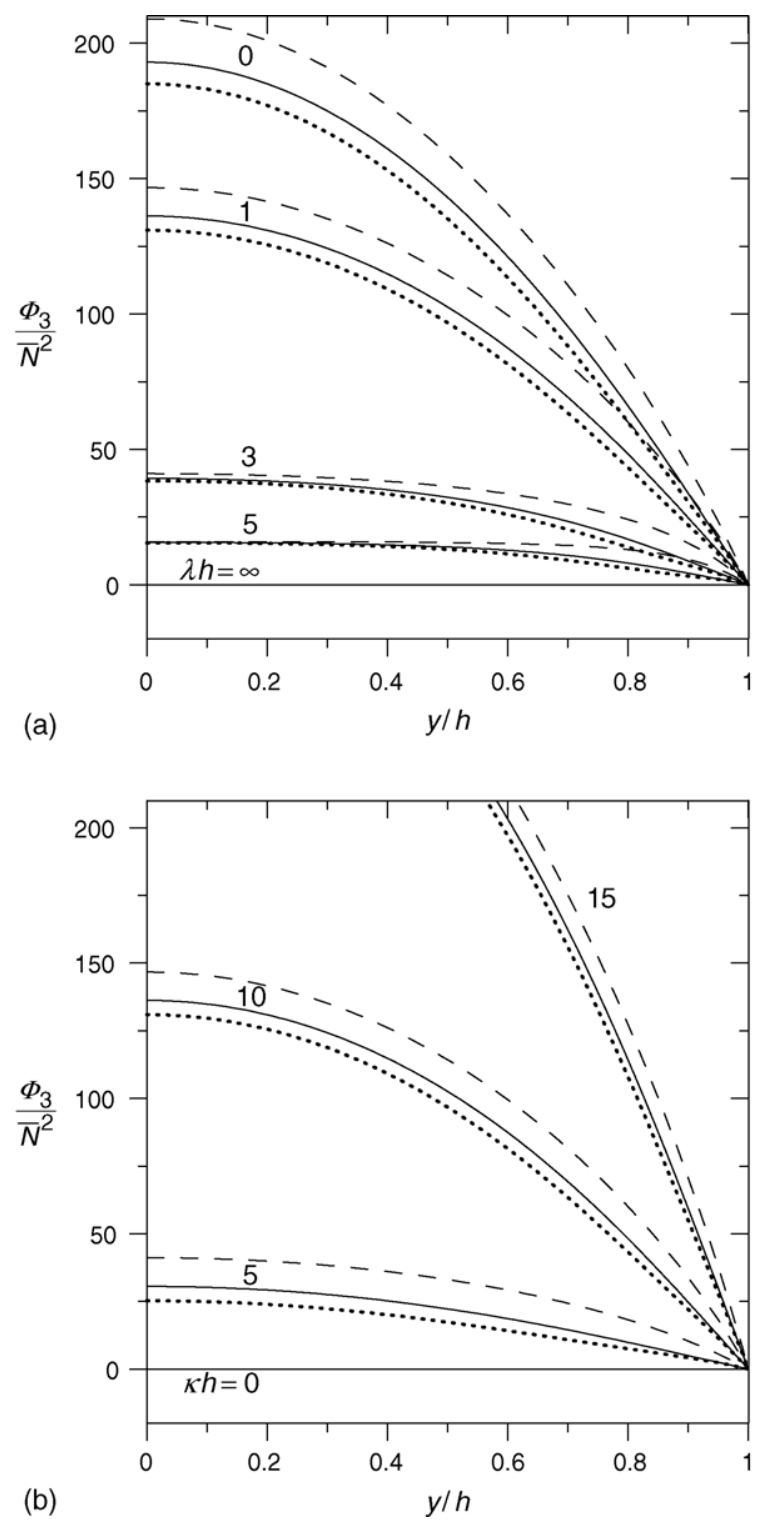

Fig. 3. Plots of the function $\Phi_{3} / \bar{N}^{2}$ for a capillary slit filled with adsorbed polyelectrolytes $(b / h=0)$ vs. the relative position $y / h$ : (a) $\kappa h=10$; (b) $\lambda h=1$. The dashed, solid, and dotted curves represent the cases $\bar{\zeta} / \bar{N}=2,0$, and -2 , respectively. 
Donnan potential everywhere, and $\bar{\sigma} / \bar{N}=0$ at any value of $\kappa h$. For the other cases with $b / h=0, \bar{\sigma}$ has the same sign as $\bar{\zeta}-\bar{N}$ and the magnitude of $\bar{\sigma} / \bar{N}$ increases monotonically with an increase in $\kappa h$ for a constant value of $\bar{\zeta} / \bar{N}$. For the case with a finite value of $b / h, \bar{\sigma} / \bar{N}$ is negative and its magnitude is still a monotonic increasing function of $\kappa h$ if $\bar{\zeta} / \bar{N} \leq 0$, but the dependence of $\bar{\sigma} / \bar{N}$ on $\kappa h$ may not be monotonic if $\bar{\zeta} / \bar{N}>0$.

The functions $\Phi_{1}, \Phi_{2}$, and $\Phi_{3}$ given by Eq. (27) for the general case and by Eqs. (31), (33), (35), and (37) for several special cases determine the diffusioosmotic velocity of a symmetric electrolyte in a capillary slit with each of its inside walls covered by a layer of adsorbed polyelectrolytes according to Eq. (26) correct to the second orders of $\bar{\sigma}$ (or
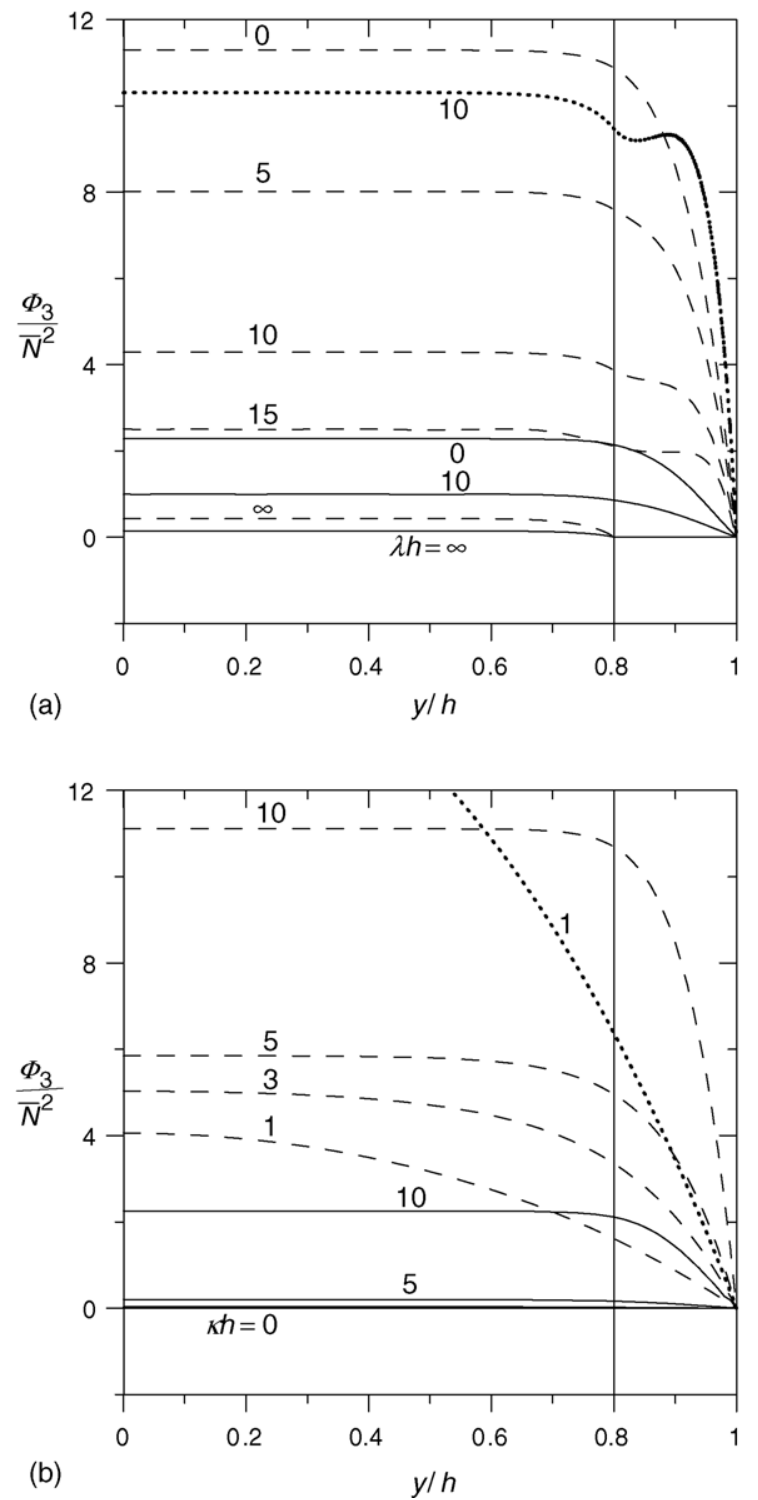

Fig. 4. Plots of the function $\Phi_{3} / \bar{N}^{2}$ for a capillary slit with its inside walls covered by layers of adsorbed polyelectrolytes vs. the relative position $y / h$ as $b / h=0.8$ : (a) $\kappa h=10$; (b) $\lambda h=1$. The solid, dashed, and dotted curves represent the cases $\bar{\zeta} / \bar{N}=0,2$, and 4 , respectively. $\bar{\zeta})$ and $\bar{N}$. Some graphical results concerning $\Phi_{1} / \bar{N}$ and $\Phi_{2} / \bar{N}^{2}$ (and Ze $\psi / k T \bar{N}$ ) as functions of the dimensionless coordinate $y / h$ can be found in the literature [22]. In Fig. 3, the function $\Phi_{3} / \bar{N}^{2}$ for a slit filled with adsorbed polyelectrolytes (with $b / h=0$ ) calculated form Eq. (37) is plotted versus $y / h$ for several values of the parameters $\bar{\zeta} / \bar{N}$, $\kappa h$, and $\lambda h$. It can be seen that $\Phi_{3}$ is positive, meaning that the effect of the lateral distribution of the induced axial electric field in the slit will cause the fluid flowing towards the end of higher electrolyte concentration. As expected, the value of $\Phi_{3}$ is a monotonically decreasing function of $y / h$ from a maximum at the median plane (with $y=0$ ) between the slit walls to zero at the no-slip walls (with $y=h$ ). The value of $\Phi_{3} / \bar{N}^{2}$ in general increases with an increase in $\bar{\zeta} / \bar{N}$. Evidently, $\Phi_{3}$
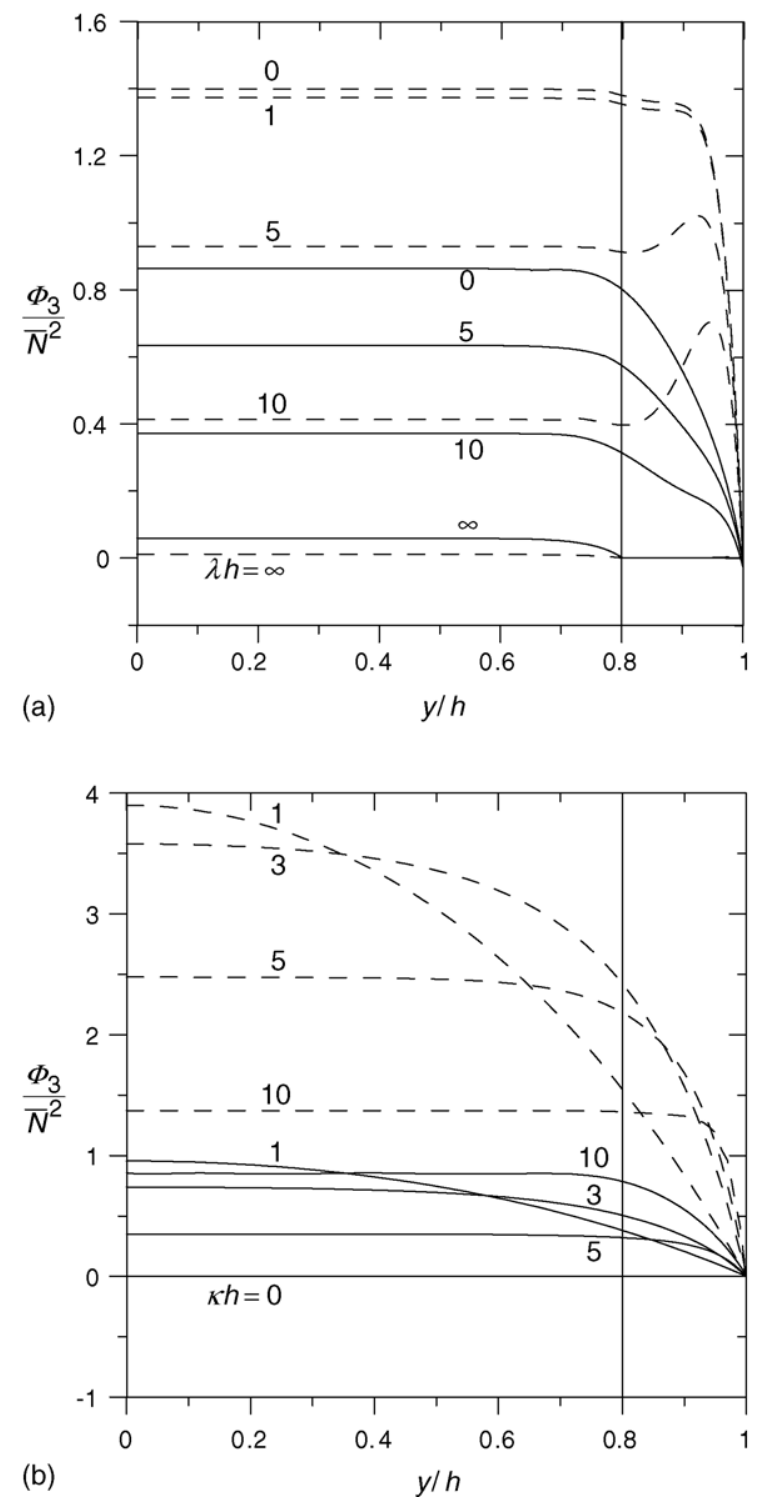

Fig. 5. Plots of the function $\Phi_{3} / \bar{N}^{2}$ for a capillary slit with its inside walls covered by layers of adsorbed polyelectrolytes vs. the relative position $y / h$ as $b / h=0.8$ : (a) $\kappa h=10$; (b) $\lambda h=1$. The solid and dashed curves represent the cases $\bar{\zeta} / \bar{N}=-1$ and -2 , respectively. 
increases with an increase in the value of $\kappa h$ and decreases with an increase in the value of $\lambda h$, for an otherwise specified condition. In the limiting situations that $\kappa h=0$ (there is no interaction between the diffuse ions and the fixed charges) or $\lambda h \rightarrow \infty$ (there is no flow penetration into the polymer layer), $\Phi_{3}$ vanishes at any position in the capillary.

The function $\Phi_{3} / \bar{N}^{2}$ for a capillary slit with its inside walls covered by finite layers of adsorbed polyelectrolytes (with $b / h=0.8$ as an example) is plotted versus the relative position $y / h$ in Figs. 4 and 5 for different values of the parameters $\bar{\zeta} / \bar{N}, \kappa h$, and $\lambda h$. Again, $\Phi_{3}$ is positive for given values of these parameters, equals zero everywhere in the capillary for the limiting case of $\kappa h=0$, and decreases with an increase in $\lambda h$ for an otherwise fixed condition. When the magnitudes of $\bar{\zeta} / \bar{N}$ and $\lambda h$ are relatively large, $\Phi_{3} / \bar{N}^{2}$ is not a monotonic function of $y / h$ due to the existence of the finite

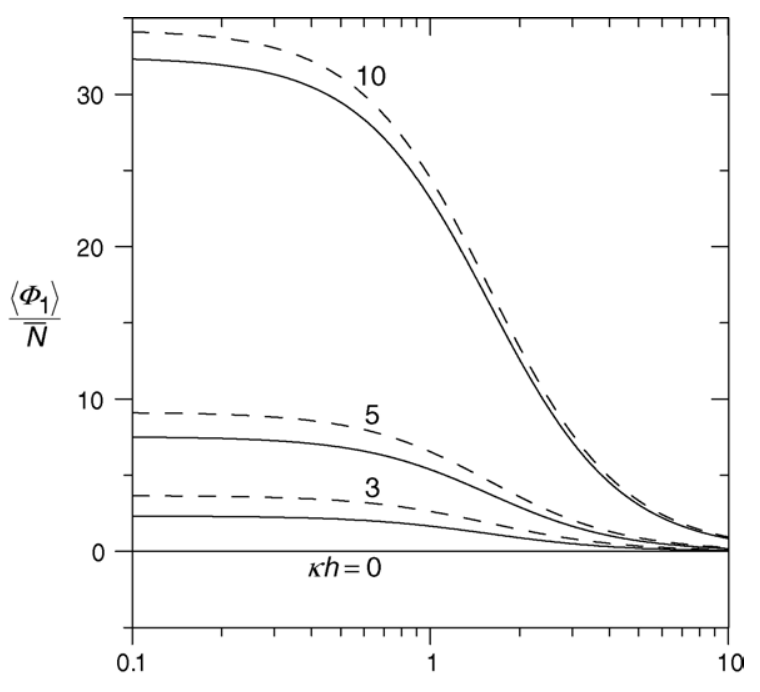

(a)

$\lambda h$

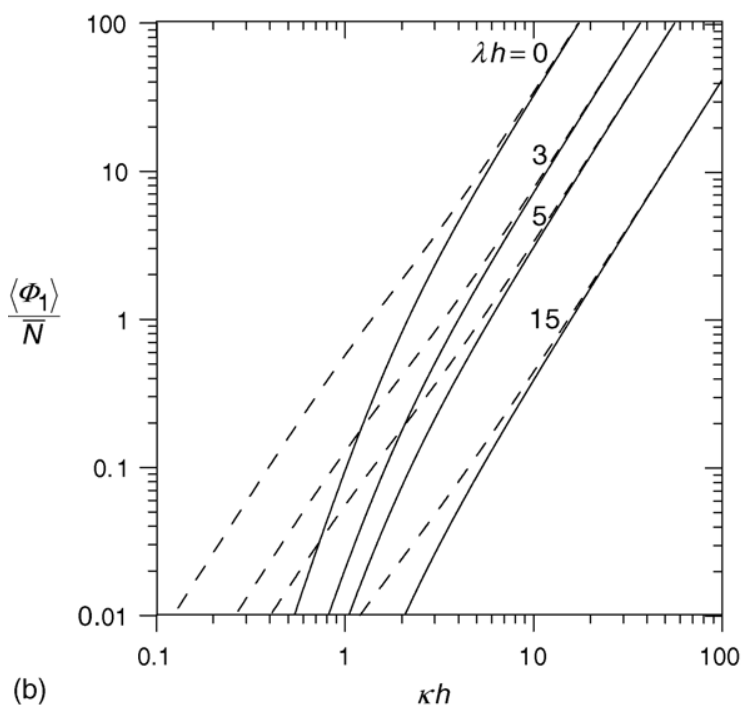

Fig. 6. Plots of the function $\left\langle\Phi_{1}\right\rangle / \bar{N}$ for a capillary slit filled with adsorbed polyelectrolytes $(b / h=0)$ vs. the parameters $\lambda h$ and $\kappa h$. The solid and dashed curves represent the cases $\bar{\zeta} / \bar{N}=0$ and 2, respectively. polyelectrolyte layers on the slit walls. In the limit $\lambda h \rightarrow \infty$, $\Phi_{3}$ vanishes within the surface charge layer $(y>b)$ as expected, but can be finite at other locations in the capillary. For the case of $\bar{\zeta} / \bar{N} \geq 0$, as illustrated in Fig. 4, the value of $\Phi_{3} / \bar{N}^{2}$ increases with an increase in $\kappa h$ or $\bar{\zeta} / \bar{N}$ for an otherwise specified condition. For the case of $\bar{\zeta} / \bar{N}<0$, as shown in Fig. 5, $\Phi_{3}$ at a given relative position not too close to the slit walls may not be a monotonically increasing function of $\kappa h$.

In Figs. 6-8, the averaged values $\left\langle\Phi_{3}\right\rangle / \bar{N}^{2},\left\langle\Phi_{2}\right\rangle / \bar{N}^{2}$, and $\left\langle\Phi_{3}\right\rangle / \bar{N}^{2}$, respectively, calculated from Eq. (38) as functions of the relevant parameters are plotted for a slit filled with adsorbed polyelectrolytes. For all cases, both $\left\langle\Phi_{2}\right\rangle / \bar{N}^{2}$ and $\left\langle\Phi_{3}\right\rangle / \bar{N}^{2}$ are positive, and the dependence of these values on the parameter $\bar{\zeta} / \bar{N}$ becomes relatively weak when the magnitude of the parameter $\kappa h$ is large. On the other hand, $\left\langle\Phi_{2}\right\rangle / \bar{N}^{2}$
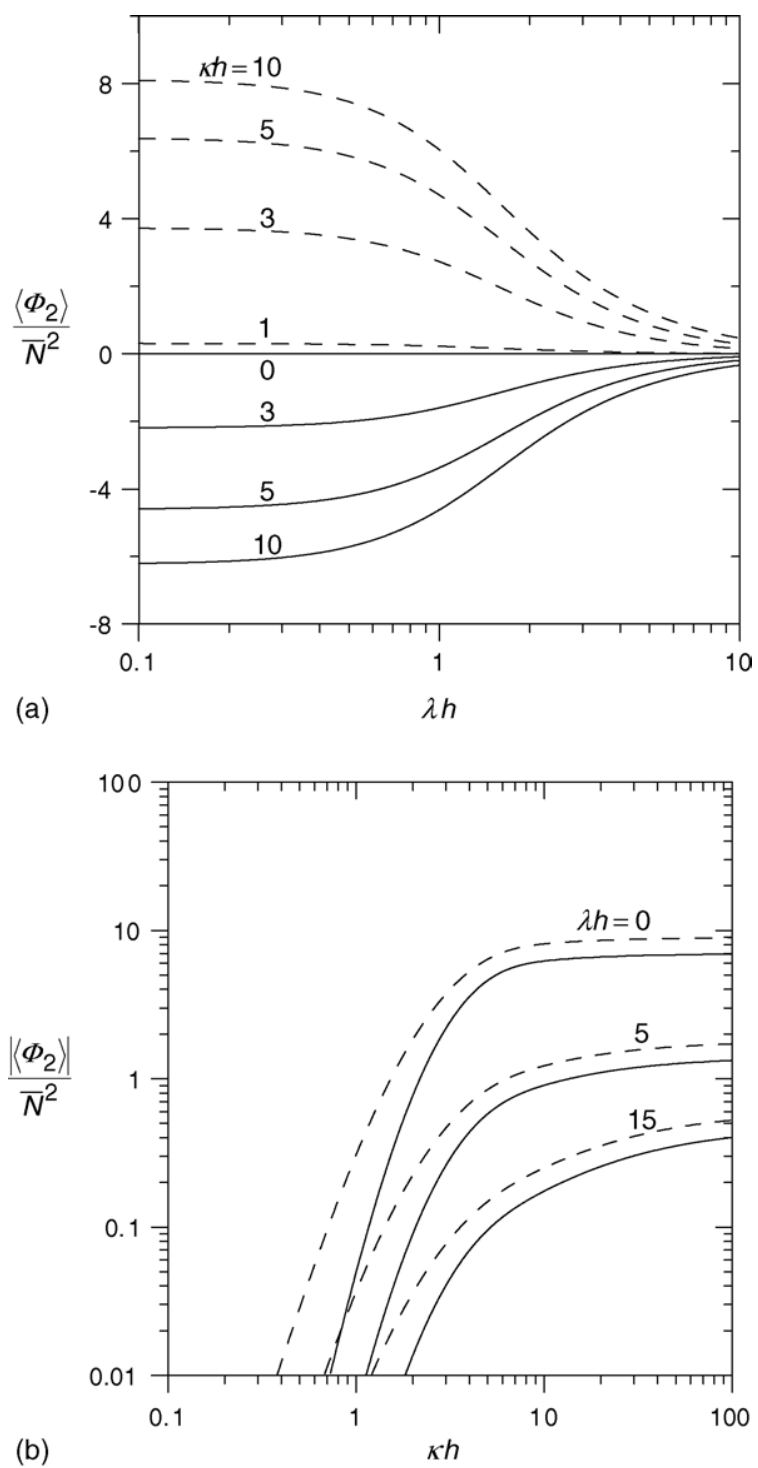

Fig. 7. Plots of the function $\left\langle\Phi_{2}\right\rangle / \bar{N}^{2}$ for a capillary slit filled with adsorbed polyelectrolytes $(b / h=0)$ vs. the parameters $\lambda h$ and $\kappa h$. The solid and dashed curves represent the cases $\bar{\zeta} / \bar{N}=0$ and 2, respectively. 
is positive as $\bar{\zeta} / \bar{N}>1$, negative as $0 \leq \bar{\zeta} / \bar{N}<1$, and vanishes as $\bar{\zeta} / \bar{N}=1$. For the case of $\bar{\zeta} / \bar{N}<0$ (which is not plotted here for conciseness), $\left\langle\Phi_{2}\right\rangle / \bar{N}^{2}$ can be either positive or negative depending on the combination of $\bar{\zeta} / \bar{N}$ and $\kappa h$. As expected, the magnitudes of all these three average functions increase with an increase in the value of $\kappa h$ (the dependence for $\left\langle\Phi_{2}\right\rangle / \bar{N}^{2}$ is weaker than for the other two functions when $\kappa h$ is large) and decrease with an increase in the value of $\lambda h$, for an otherwise specified condition. In the limiting situations that $\kappa h=0$ or $\lambda h \rightarrow \infty,\left\langle\Phi_{1}\right\rangle=\left\langle\Phi_{2}\right\rangle=\left\langle\Phi_{3}\right\rangle=0$ and there is no fluid flow in the capillary.

The dependence of the normalized average diffusioosmotic velocity $\langle u\rangle / U^{*}$ of an electrolyte solution in a capillary slit with each of its inside walls covered by a layer of adsorbed polyelectrolytes on the dimensionless fixed-charge density
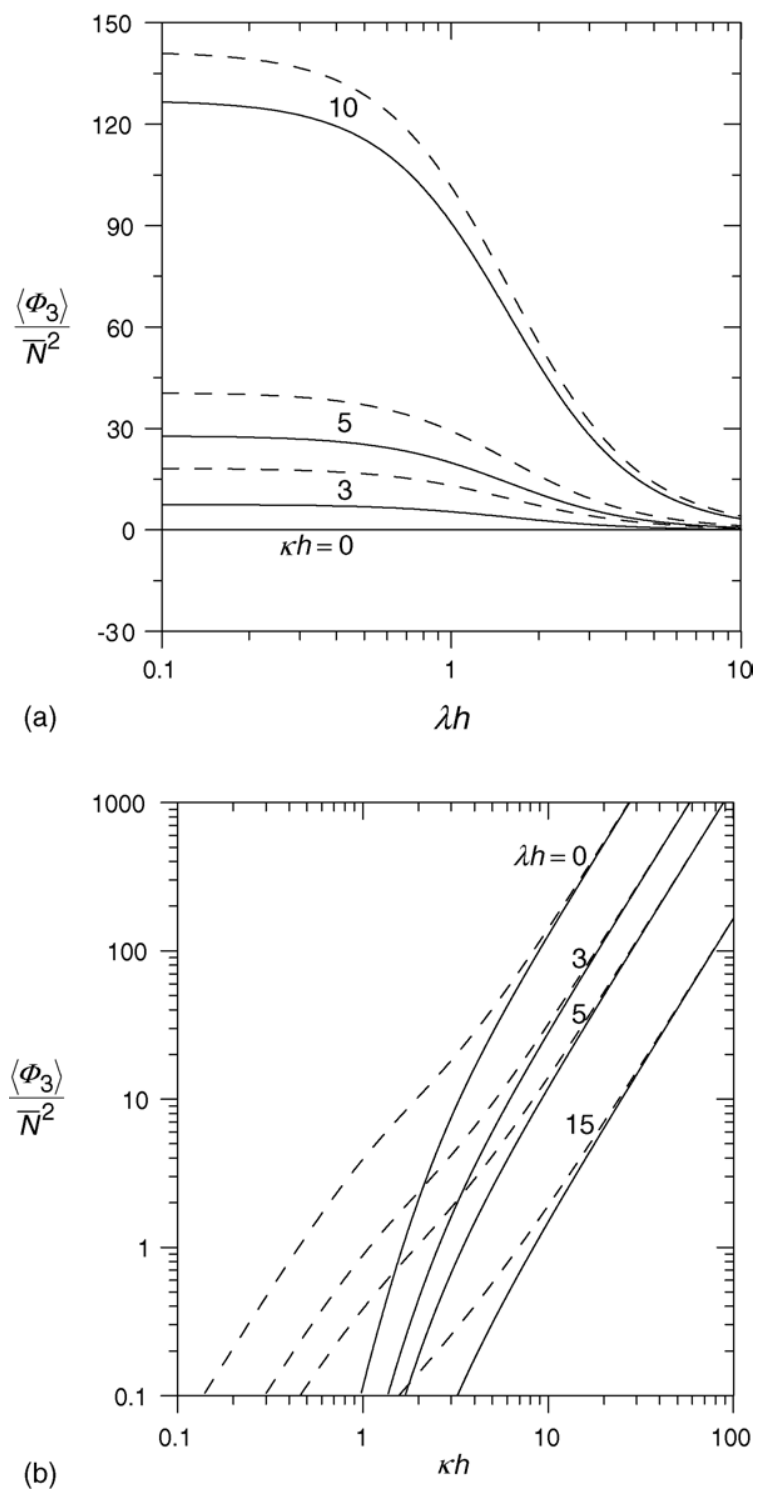

Fig. 8. Plots of the function $\left\langle\Phi_{3}\right\rangle / \bar{N}^{2}$ for a capillary slit filled with adsorbed polyelectrolytes $(b / h=0)$ vs. the parameters $\lambda h$ and $\kappa h$. The solid and dashed curves represent the cases $\bar{\zeta} / \bar{N}=0$ and 2, respectively.
$\bar{N}$ at a fixed value of $\kappa h$ and various values of $\bar{\zeta} / \bar{N}, \lambda h$, and $b / h$ calculated from Eqs. (29) and (30) is displayed in Figs. 9 and 10. Because our analysis is based on the assumption of small electrostatic potentials, the magnitudes of $\bar{N}$ considered are less than 3. Fig. 9 is drawn for the case of a symmetric electrolyte that the cation and anion diffusivities are equal $(\beta=0$, representing the aqueous solution of $\mathrm{KCl}$ if $Z=1$ ). Only the results at positive values of $\bar{N}$ are shown because the fluid velocity, which is due to $\mathrm{O}\left(\zeta^{2}, \zeta N, N^{2}\right)$ contribution entirely as illustrated by Eqs. (29) and (30), is now an even function of $\bar{N}$. It can be seen from Eq. (29) and Figs. 7 and 8 that the fluid flows contributed from chemiosmosis (involving the function $\Phi_{2}$ ) and electroosmosis (involving
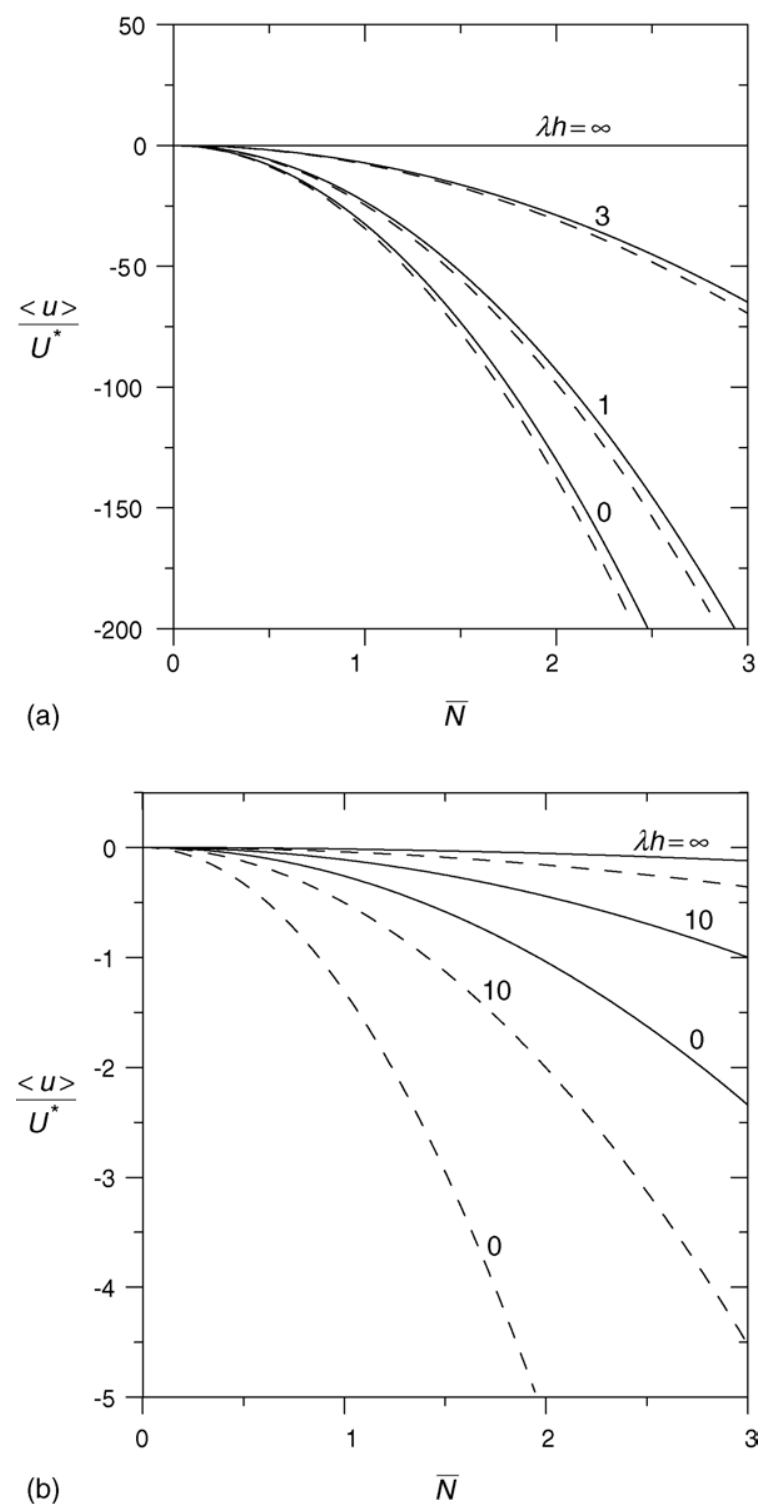

Fig. 9. Plots of the normalized average diffusioosmotic velocity $\langle u\rangle / U^{*}$ in a capillary slit with its inside walls covered by layers of adsorbed polyelectrolytes vs. the dimensionless charge density $\bar{N}$ with $\kappa h=10$ and $\beta=0$ : (a) $b / h=0$; (b) $b / h=0.8$. The solid and dashed curves represent the cases $\bar{\zeta} / \bar{N}=0$ and 2 , respectively. 

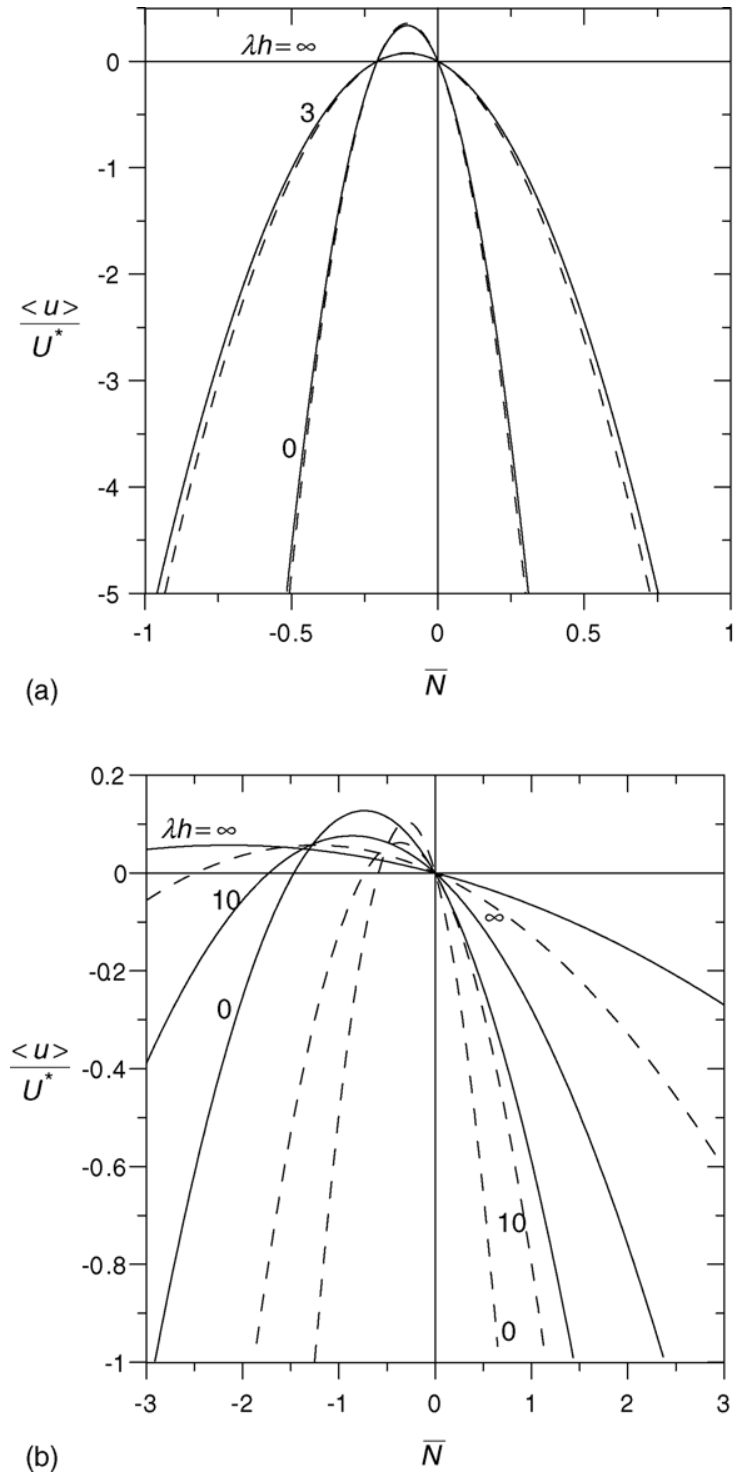

Fig. 10. Plots of the normalized average diffusioosmotic velocity $\langle u\rangle / U^{*}$ in a capillary slit with its inside walls covered by layers of adsorbed polyelectrolytes vs. the dimensionless charge density $\bar{N}$ with $\kappa h=10$ and $\beta=-0.2$ : (a) $b / h=0$; (b) $b / h=0.8$. The solid and dashed curves represent the cases $\bar{\zeta} / \bar{N}=0$ and 2 , respectively.

$\Phi_{3}$ ) are in the same direction as $0 \leq \bar{\zeta} / \bar{N}<1$ but in the opposite directions as $\bar{\zeta} / \bar{N}>1$, and the net flow is dominated by the electroosmotic effect (having the direction of increasing electrolyte concentration). As expected, the magnitude of $\langle u\rangle / U^{*}$ increases monotonically with an increase in $|\bar{N}|$ and with a decrease in $\lambda h$ for constant values of $\bar{\zeta} / \bar{N}, \kappa h$, and $b / h$. There is no diffusioosmotic motion of the fluid for the special case of $\bar{\zeta}=\bar{N}=0$. In a previous study of the same diffusioosmosis [22], the $\mathrm{O}\left(\zeta^{2}, \zeta N, N^{2}\right)$ contribution from electroosmotic effect was not considered, and the resulted fluid velocity (which is due to chemiosmotic effect only) for the case of $\bar{\zeta} / \bar{N}>1$ was in the direction of decreasing electrolyte concentration. Note that the magnitude of $\langle u\rangle / U^{*}$ has the order unity for the case of a thin polyelectrolyte layer $(b / h \rightarrow 1)$, but can be quite large if $b / h \rightarrow 0$.

In Fig. 10, the normalized average diffusioosmotic velocity $\langle u\rangle / U^{*}$ as a function of $\bar{N}$ is plotted for the case of a symmetric electrolyte whose cation and anion have different diffusion coefficients ( $\beta=-0.2$ is chosen, representing the aqueous solution of $\mathrm{NaCl}$ if $Z=1$ ). In this case, the diffusioosmotic velocity is neither an even nor an odd function of $\bar{N}$. It can be seen that the fluid velocity is not necessarily a monotonic function of the magnitude of $\bar{N}$ for fixed values of $\bar{\zeta} / \bar{N}, \kappa h, \lambda h$, and $b / h$. The curves in the vicinity of $\bar{N}=0$ indicate that the fluid might reverse direction of flow more than once as $\bar{N}$ varies in a narrow range from negative to positive values. The reversals occurring at the values of $\bar{N}$ other than zero result from the combination of the contributions from electroosmosis of $\mathrm{O}(\zeta, N)$ and $\mathrm{O}\left(\zeta^{2}, \zeta N, N^{2}\right.$ ) (which is still dominant in many situations) and chemiosmosis of $\mathrm{O}\left(\zeta^{2}, \zeta N, N^{2}\right)$. Again, for specified values of $\bar{\zeta}, \bar{N}, \kappa h$, and $b / h$, the magnitude of $\langle u\rangle / U^{*}$ decreases monotonically with an increase in $\lambda h$. In general, the net diffusioosmotic flow is still dominated by the electroosmotic contribution and in the direction of increasing electrolyte concentration.

\section{Concluding remarks}

The steady diffusioosmotic flow of solutions of symmetric electrolytes in a narrow capillary slit bearing a layer of adsorbed polyelectrolytes on each of its inside walls is analytically studied in this work. Solving the linearized Poisson-Boltzmann equation and the modified Navier-Stokes/Brinkman equation applicable to the system, the electrostatic potential distribution and the fluid velocity profile under the influence of a constant gradient of the electrolyte concentration are obtained in closed forms to the orders $(\zeta, N)$ and $\left(\zeta^{2}, \zeta N, \bar{N}^{2}\right)$, respectively. The macroscopic electric field induced by the prescribed electrolyte concentration gradient through the capillary slit is a function of the lateral position rather than a constant bulk-phase quantity. The contribution to the diffusioosmotic flow made by the position dependence of the induced electric field is of the same order $\left[\mathrm{O}\left(\zeta^{2}, \zeta N, N^{2}\right)\right]$ as, but may have an opposite direction to, that made by the chemiosmotic effect, and the former is dominant in most practical situations, as indicated by Eq. (26). Therefore, the effect of the deviation of the local induced axial electric field in the slit from its bulk-phase quantity [given by the term of zeroth order in $\psi$ in Eq. (19)], which causes the fluid flowing towards the end of higher electrolyte concentration, cannot be neglected in the evaluation of the diffusioosmotic flow rate in a capillary, even for the case of very thin double layer. Our results demonstrate that the structure of the surface charge layer can lead to a quite different diffusioosmotic flow from that in a capillary with bare walls [16], depending on the characteristics of the capillary, of the surface charge layer, and of the electrolyte solution. 
The macroscopic electric field $\boldsymbol{E}$ arising spontaneously due to the imposed concentration gradient of the electrolyte in the axial direction of the capillary slit is provided by Eqs. (15) or (19), and the diffusioosmotic velocity $u$ of the electrolyte solution is obtained in Eq. (26); both are accurate with the primary effects only. In addition to the ionic fluxes due to diffusion and electric migration [given by Eq. (14)], the diffusioosmotic fluid flow can generate an electric current by ionic convection (known as the relaxation effect). This electric current is not included in the current balance for the determination of $\boldsymbol{E}$ in Section 3. Thus, a secondary induced electric field must build up through the capillary, which is just sufficient to prevent the net electric current flow. This secondary electric field, which is of the order $\mathrm{O}\left(\zeta^{2}, \zeta N, N^{2}\right)$, and its contribution to the fluid flow, which is of the order $\mathrm{O}\left(\zeta^{3}, \zeta^{2} N, \zeta N^{2}, N^{3}\right)$, can be calculated via a similar approach in the calculations of the streaming potential induced across the capillary in the presence of an applied pressure gradient [2,3,21]. Alternatively, distributions of ionic concentrations, electric potentials, and fluid velocity might be calculated in a general self-consistent way $[13-15,28]$. For the geometry of a capillary slit with adsorbed polyelectrolyte layers considered in the present article, however, the general approach accurate to higher orders of $\zeta$ and $N$ would be somewhat complicated and could be an endeavor for future work.

\section{Acknowledgment}

This research was supported by the National Science Council of the Republic of China.

\section{Appendix A}

For conciseness the definitions of some functions in Section 4 are listed here. In Eq. (27),

$$
\begin{aligned}
& g_{10}(x)=\frac{\kappa}{\lambda} {\left[\frac{\kappa^{2}}{\kappa^{2}-\lambda^{2}} F_{2}(x)-A \sinh x\right], } \\
& g_{20}(x)=\frac{\kappa}{\lambda}\left[\frac{4 \kappa^{2}}{4 \kappa^{2}-\lambda^{2}} F_{4}(x)+\frac{8 \kappa^{2}}{\kappa^{2}-\lambda^{2}} \bar{N} F_{2}(x)\right. \\
&\left.-A^{2}(\sinh 2 x-2 x)\right], \\
& g_{30}(x)=\frac{\kappa}{\lambda}\left[\frac{4 \kappa^{2}}{4 \kappa^{2}-\lambda^{2}} F_{4}(x)+\frac{8 \kappa^{2}}{\kappa^{2}-\lambda^{2}} \bar{N} F_{2}(x)\right. \\
&\left.-A^{2}(\sinh 2 x+2 x)\right] ;
\end{aligned}
$$

$g_{11}(x)=A \cosh x$,

$$
\begin{aligned}
g_{21}(x)= & \frac{1}{2} A^{2}\left(\cosh 2 x-2 x^{2}\right), \\
g_{31}(x)= & \frac{1}{2} A^{2}\left(\cosh 2 x+2 x^{2}\right) ; \\
g_{12}(x)= & \frac{\kappa^{2}}{\kappa^{2}-\lambda^{2}} F_{1}(x)-\frac{\kappa^{2}}{\lambda^{2}} \bar{N}, \\
g_{22}(x)= & \frac{2 \kappa^{2}}{4 \kappa^{2}-\lambda^{2}} F_{3}(x)+\frac{8 \kappa^{2}}{\kappa^{2}-\lambda^{2}} \bar{N} F_{1}(x) \\
& +\frac{2 \kappa^{2}}{\lambda^{2}}\left(2 A^{2}-B^{2}+C^{2}-2 \bar{N}^{2}\right), \\
g_{32}(x)= & g_{22}(x)-\frac{4 \kappa^{2}}{\lambda^{2}} A^{2},
\end{aligned}
$$

where

$$
\begin{aligned}
& F_{1}(x)=B \cosh x+C \sinh x, \\
& F_{2}(x)=(B \sinh x+C \cosh x), \\
& F_{3}(x)=\left(B^{2}+C^{2}\right) \cosh 2 x+2 B C \sinh 2 x, \\
& F_{4}(x)=\left(B^{2}+C^{2}\right) \sinh 2 x+2 B C \cosh 2 x .
\end{aligned}
$$

In Eq. (30),

$$
\begin{aligned}
& s_{11}(x)=A \frac{\sinh x}{\kappa h} \\
& s_{21}(x)=\frac{1}{12 \kappa h} A^{2}\left(3 \sinh 2 x-4 x^{3}\right), \\
& s_{31}(x)=\frac{1}{12 \kappa h} A^{2}\left(3 \sinh 2 x+4 x^{3}\right) ; \\
& s_{12}(x)=\frac{1}{\kappa h} \frac{\kappa^{2}}{\kappa^{2}-\lambda^{2}}\left[F_{2}(\kappa h)-F_{2}(x)\right]-\frac{\kappa}{\lambda^{2} h} \bar{N}(\kappa h-x),
\end{aligned}
$$

$$
\begin{aligned}
s_{22}(x)= & \frac{1}{\kappa h} \frac{\kappa^{2}}{4 \kappa^{2}-\lambda^{2}}\left[F_{4}(\kappa h)-F_{4}(x)\right] \\
& +\frac{8}{\kappa h} \frac{\kappa^{2}}{\kappa^{2}-\lambda^{2}} \bar{N}\left[F_{2}(\kappa h)-F_{2}(x)\right] \\
& +\frac{2 \kappa}{\lambda^{2} h}\left(2 A^{2}-B^{2}+C^{2}-2 \bar{N}^{2}\right)(\kappa h-x),
\end{aligned}
$$

$s_{32}(x)=s_{22}(x)-\frac{4 \kappa}{\lambda^{2} h} A^{2}(\kappa h-x)$.

In Eqs. (35) and (36),

$$
\begin{aligned}
& g_{13}(x)=F_{1}(\kappa h)-F_{1}(x), \\
& g_{23}(x)=g_{33}(x)=\frac{1}{2}\left[F_{3}(\kappa h)-F_{3}(x)\right]+8 \bar{N} g_{13}(x) ; \\
& s_{13}(x)=\frac{1}{\kappa h}\left[F_{1}(\kappa h)(\kappa h-x)-F_{2}(\kappa h)+F_{2}(x)\right],
\end{aligned}
$$




$$
\begin{aligned}
s_{23}(x)= & s_{33}(x)=\frac{1}{2 \kappa h} F_{3}(\kappa h)(\kappa h-x) \\
& -\frac{1}{4 \kappa h}\left[F_{4}(\kappa h)-F_{4}(x)\right]+8 \bar{N} s_{13}(x) .
\end{aligned}
$$

In Eqs. (37) and (38),

$$
\begin{aligned}
g_{14}(x)= & \frac{\kappa^{2}}{\kappa^{2}-\lambda^{2}} B_{1} \cosh x-\frac{\kappa^{2}}{\lambda^{2}} \bar{N} \\
g_{24}(x)= & \frac{2 \kappa^{2}}{4 \kappa^{2}-\lambda^{2}} B_{1}^{2} \cosh 2 x+\frac{8 \kappa^{2}}{\kappa^{2}-\lambda^{2}} B_{1} \bar{N} \cosh x \\
& +\frac{2 \kappa^{2}}{\lambda^{2}}\left(B_{1}^{2}+4 B_{1} \bar{N}\right) \\
g_{34}(x)= & g_{24}(x)-\frac{4 \kappa^{2}}{\lambda^{2}}\left(B_{1}+\bar{N}\right)^{2} ; \\
s_{14}(x)= & \frac{\kappa^{2}}{\kappa^{2}-\lambda^{2}} B_{1} \frac{\sinh ^{2}}{\kappa h}-\frac{\kappa x}{\lambda^{2} h} \bar{N}, \\
s_{24}(x)= & \frac{\kappa^{2}}{4 \kappa^{2}-\lambda^{2}} B_{1}^{2} \frac{\sinh 2 x}{\kappa h}+\frac{8 \kappa^{2}}{\kappa^{2}-\lambda^{2}} B_{1} \bar{N} \frac{\sinh x}{\kappa h} \\
& +\frac{2 \kappa x}{\lambda^{2} h}\left(B_{1}{ }^{2}+4 B_{1} \bar{N}\right), \\
s_{34}(x)= & s_{24}(x)-\frac{4 \kappa x}{\lambda^{2} h}\left(B_{1}+\bar{N}\right)^{2}
\end{aligned}
$$

and $B_{1}$ was defined by Eq. (13).

\section{References}

[1] M. Smoluchowski, in: I. Graetz (Ed.), Handbuch der Electrizitat und des Magnetismus, vol. II, Barth, Leipzig, 1921, p. 336.
[2] D. Burgreen, F.R. Nakache, J. Phys. Chem. 68 (1964) 1084.

[3] C.L. Rice, R. Whitehead, J. Phys. Chem. 69 (1965) 4017.

[4] S.S. Dukhin, B.V. Derjaguin, in: E. Matijevic (Ed.), Surface and Colloid Science, vol. 7, Wiley, New York, 1974.

[5] J.L. Anderson, K. Idol, Chem. Eng. Commun. 38 (1985) 93.

[6] J.H. Masliyah, Electrokinetic Transport Phenomena, AOSTRA, Edmonton, Alta., Canada, 1994.

[7] A. Szymczyk, B. Aoubiza, P. Fievet, J. Pagetti, J. Colloid Interface Sci. 216 (1999) 285.

[8] H.J. Keh, H.C. Tseng, J. Colloid Interface Sci. 242 (2001) 450.

[9] D.C. Prieve, Adv. Colloid Interface Sci. 16 (1982) 321.

[10] D.C. Prieve, J.L. Anderson, J.P. Ebel, M.E. Lowell, J. Fluid Mech. 148 (1984) 247.

[11] J.L. Anderson, Annu. Rev. Fluid Mech. 21 (1989) 61.

[12] H.J. Keh, S.B. Chen, Langmuir 9 (1993) 1142.

[13] J.C. Fair, J.F. Osterle, J. Chem. Phys. 54 (1971) 3007.

[14] V. Sasidhar, E. Ruckenstein, J. Colloid Interface Sci. 85 (1982) 332.

[15] G.B. Westermann-Clark, J.L. Anderson, J. Electrochem. Soc. 130 (1983) 839.

[16] H.J. Keh, H.C. Ma, Colloid Surf. A 233 (2004) 87.

[17] E. Donath, A. Voigt, J. Colloid Interface Sci. 109 (1986) 122.

[18] H. Ohshima, T. Kondo, J. Colloid Interface Sci. 135 (1990) 443.

[19] V.M. Starov, Y.E. Solomentsev, J. Colloid Interface Sci. 158 (1993) 166.

[20] H.J. Keh, Y.C. Liu, J. Colloid Interface Sci. 172 (1995) 222.

[21] H.J. Keh, J.M. Ding, J. Colloid Interface Sci. 263 (2003) 645.

[22] J.H. Wu, H.J. Keh, Colloid Surf. A 212 (2003) 27.

[23] K.A. Sharp, D.E. Brooks, Biophys. J. 47 (1985) 563.

[24] K. Morita, N. Muramatsu, H. Ohshima, T. Kondo, J. Colloid Interface Sci. 147 (1991) 457.

[25] O. Aoyanagi, N. Muramatsu, H. Ohshima, T. Kondo, J. Colloid Interface Sci. 162 (1994) 222.

[26] S.S. Dukhin, R. Zimmermann, C. Werner, J. Colloid Interface Sci. 274 (2004) 309.

[27] A.W. Adamson, Physical Chemistry of Surfaces, fifth ed., Wiley, New York, 1990.

[28] V.M. Starov, W.R. Bowen, J.S. Welfoot, J. Colloid Interface Sci. 240 (2001) 509. 